\title{
Termografía, una herramienta novedosa en la práctica estomatológica
}

\author{
Thermography, a novel tool in stomatological practice
}

Lianne Laura de León Ramírez. ', María Isabel Garay Crespo. ${ }^{2}$, Layma Rosa de León Ramírez. ${ }^{3} \&$ Lia Magariño Abreus. ${ }^{4}$

\begin{abstract}
Introduction: Thermography is a technique that allows to determine temperatures from a distance without the need for physical contact with the object to be studied, in this sense it has become a very useful complementary tool for the evaluation of painful processes, allowing to identify pathophysiological alterations in the people. Its medical applications are multiple and dentistry does not escape its field of application.
\end{abstract}

Objective: to expose the applications of

\section{Resumen}

Introducción: la Termografía es una técnica que permite determinar temperaturas a distancia sin necesidad de contacto físico con el objeto a estudiar, en este sentido se ha convertido en una herramienta complementaria muy útil para la evaluación de los procesos dolorosos, permitiendo identificar alteraciones fisiopatológicas en las personas. Sus aplicaciones médicas son múltiples y la odontología no escapa de su campo de aplicación.

1 Universidad de Ciencias Médicas de Matanzas, Matanzas, Cuba, Estudiante de Tercer Año de Estomatología, liannelaura@nauta.cu_(D_http://orcid.org/0000-0001-9250-1889

${ }^{2}$ Universidad de Ciencias Médicas de Matanzas, Matanzas, Cuba, Especialista en Segundo Grado en Estomatología General Integral, mtz0116@infomed.sld.cu_ (D https://orcid.org/0000-0001-9406-1637

${ }^{3}$ Universidad de Ciencias Médicas de Matanzas, Matanzas, Cuba, Especialista en Primer Grado en Medicina General Integral, laymarosadeleonramirez@gmail.com (D) https://orcid.org/0000-0002-0470-9207

${ }^{4}$ Universidad de Ciencias Médicas de Cienfuegos, Cienfuegos, Cuba, Estudiante de Tercer Año de Estomatología, lya.magarino2000@gmail.com (D) https://orcid.org/0000-0002-9933-5323 
Thermography in Stomatology.

Method: a bibliographic review was carried out, using the resources available in the Infomed network, specifically: PubMed, SciELO and Ebesco, through different databases (Medline, Academic Search Premier, Medic Latina) in addition to Cumed, Lilacs and Scopus. Searches were conducted in English and Spanish. The Google Scholar search engine was used.

Results: they refer to conditions in which this technique is used to establish a diagnosis. Thermography is a useful option to complement the diagnosis of certain oral pathologies that involve a change in temperature in the orofacial region, and it can affect people's health as part of the health-disease process.

Keywords: thermography, thermology, temperature, thermography in stomatology.
Objetivo: exponer las aplicaciones de la Termografía en la Estomatología.

Método: se realizó una revisión bibliográfica, empleando los recursos disponibles en la red Infomed, específicamente: PubMed, SciELO y Ebesco, a través de diferentes bases de datos (Medline, Academic Search Premier, Medic Latina) además de Cumed, Lilacs y Scopus. Las búsquedas se realizaron en inglés y español. Se utilizó el buscador Google Académico.

Resultados: se refieren afecciones en las que esta técnica es utilizada para establecer un diagnóstico. La termografía es una opción útil para complementar el diagnóstico de ciertas patologías bucales que implican un cambio de temperatura en la región orofacial, y puede incidir en la salud de las personas como parte del proceso salud-enfermedad.

Palabras claves: termografía, termología, temperatura, termografía en estomatología.

\section{Introducción}

El cuerpo humano realiza constantemente intercambios de temperatura con el entorno en forma de energía, como parte de un proceso de autorregulación para mantener la homeostasis que necesita. Esta cantidad de energía crece y decrece en proporción a la temperatura corporal, de forma que, a mayor temperatura, mayor intensidad de energía infrarroja irradiada por el cuerpo. (Salazar, Domínguez, Iborra, de Córdoba \&Gómez, 2015)

La temperatura es esencial para el desarrollo normal del cuerpo humano. Un cambio en unos pocos grados en la temperatura central del cuerpo, es un indicador de probable enfermedad. Como la circulación sanguínea difiere en varias partes de la piel, la temperatura cambia en consecuencia. Por lo tanto, las enfermedades afectan el flujo sanguíneo y causan irregularidades en la distribución de esta temperatura. (Lakshmi, Kiran\&Joy, 2018)

La piel es la ruta principal para la disipación de calor usando la sangre como fluido de intercambio de calor ya que los procesos bioquímicos en el cuerpo humano generan calor, que debe ser disipado. La temperatura de la piel es un indicador de aberraciones en el 
metabolismo, la hemodinámica o la termorregulación neuronal. (Sikdar, Khandelwal, Ghom, Diwan\&Debta, 2010)

Durante siglos, los antiguos médicos griegos describieron la relación entre el calor y los procesos patológicos. Por ejemplo, Hipócrates usó la parte posterior de su mano para sentir el calor irradiado por las partes del cuerpo que estaban siendo examinados. Así nació la termografía. Con los años, el termómetro de mercurio, creado Boullain basado en la concepción teórica creado por Sanctorius, destinado a registrar cambios temperatura corporal, fue evolucionando. (Presídeo, Wanerly\& Medrano, 2016)

Carl Wunderlich fue el primer médico que dedicó su investigación a los cambios de la temperatura humanos, midiendo las diferencias entre sanos y enfermos durante años con un termómetro donde apreciaba las variaciones en torno a los $37^{\circ} \mathrm{C}$ que experimenta el cuerpo. (Ring, 2007) La era moderna de la teletermometría fue iniciado en 1931 por Hardy, quien describió la fisiología papel de la emisión infrarroja de la piel humana y su potencial importancia diagnóstica. En 1986, la Academia Estadounidense de la Termografía Neuromuscular publicó pautas básicas para realización de exámenes termográficos faciales. (Mouli et al.,2012)

La termografía es una técnica proveniente de la física que la medicina ha aprovechado para conocer mejor los cambios fisiológicos del cuerpo humanos. El fundamento del uso en que se basa la técnica reside en que bajo los cambios de temperatura existen condiciones médicas particulares, vinculadas a la existencia de patologías. (Salazar et al., 2015)

Tiene aplicación en numerosas disciplinas como lo son: la industria civil y militar, la agrónoma, la medicina forense, la psicología, en campos de la medicina como neurología, cardiología, reumatología, dermatología, oncología, neonatos, oftalmología y cirugía, pero como tal lo que nos interesa es su aplicación en la relación entre la temperatura humana y la enfermedad, que se lleva investigando desde que se estudia la medicina. (Ring, 2007), (Salazar et al., 2015) Como se puede apreciar, sus aplicaciones médicas son múltiples y la odontología no escapa de su campo de aplicación.

Por la novedad que representa y sus múltiples funciones se decidió hacer una actualización del tema, con el objetivo de exponer las aplicaciones de la Termografía en la especialidad estomatológica. Constituye un reto para la mejora de la atención a nuestros pacientes, por lo cual resultaría interesante que los estudiantes de las universidades médicas manejen el conocimiento de las tecnologías actuales utilizadas en el campo de la salud.

\section{Metodología}

Se realizó una revisión bibliográfica, empleando los recursos disponibles en la red Infomed, específicamente: PubMed, SciELO y Ebesco, a través de las bases de datos: Medline, Academic Search Premier, Medic Latina; además de Cumed, Lilacs y Scopus. Las búsquedas se realizaron en inglés y español. Se utilizó el buscador Google Académico 
y se aplicó una estrategia de búsqueda utilizando las palabras claves y conectores: termografía, termología, temperatura, termografía en estomatología (filtrado por relevancia). Se analizó la calidad, fiabilidad y validez metodológica de los artículos seleccionados para realizar una adecuada revisión y se valoraron las tendencias actuales en la temática.

\section{Resultados}

La termografía es una técnica que permite medir temperaturas a distancia, con exactitud y sin necesidad de contacto físico con el objeto a estudiar. La cámara térmica o infrarroja que se usa para su aplicación, es capaz de captar diferencias de temperatura mínimas y convertirlas en una imagen térmica nítida en la que se pueden observar detallados sus valores exactos. (Salazar et al., 2015)

Dependiendo del tipo de operación, la termografía se puede clasificar de la siguiente manera:

1. Método de contacto: termografía de cristal líquido (LCT).

2. Método sin contacto: teletermografía infrarroja (ITT) y Teletermometría de área dinámica (DAT). (Lakshmi et al., 2018)

La termografía infrarroja (TIR) se ha convertido en una herramienta complementaria muy útil para la evaluación de los procesos dolorosos, permitiendo identificar alteraciones fisiopatológicas del medio interno que justifican la clínica dolorosa manifestada por los pacientes. Constituye una herramienta sencilla, biosostenible y de bajo coste, que no utiliza radiaciones de ningún tipo y puede utilizarse en niños y embarazadas sin riesgo alguno. (Hidalgo, Álvarez \& Salvador, 2014)

La radiación infrarroja es la señal de entrada que la cámara termográfica necesita para generar una imagen de un espectro de colores, en el que cada uno de los colores, según una escala, significa una temperatura distinta, de manera que la temperatura medida más elevada aparece en color blanco. (Salazar et al., 2015)

Dependiendo de la determinación de la temperatura de la piel se clasifica en:

1. Termografía estática: medición de temperatura en una sola instancia de tiempo.

2. Termografía dinámica: temperatura medida en serie de muchas instancias consecutivas. (Lakshmi et al., 2018)

La técnica proporciona una evaluación del dolor agudo y crónico a través de la imagen que refleja el aumento y la disminución de la temperatura debido a microcirculación en la región afectada. Ella ha contribuido para el diagnóstico de diversas enfermedades como dermatitis, trastornos sanguíneos, daño nervioso agentes sensibles y procesos inflamatorios subyacentes. En particular, en el contexto de la Odontología, esta técnica se ha utilizado en para establecer y confirmar posibles diagnósticos por ejemplo dolor miofascial, disfunción 
temporo-mandibular, cambios nerviosos, dolor de muelas enfermedad atípica, herpes labial prodrómico, peri-operatorio-dentales y endodoncia. (Presídeo et al.,2016)

Aplicación clínica de la termografía.

Las diferentes actividades de los tejidos y órganos del cuerpo afectan la temperatura corporal y determinadas enfermedades pueden mostrar patrones térmicos característicos. En condiciones normales, nuestro cuerpo presenta un termograma simétrico, basado en la inervación de los diferentes miembros por lo que emitimos el mismo calor con lugares simétricos de nuestro cuerpo. (Brioschi,2006) Cuando se altera esa simetría térmica al menos medio grado, algo está pasando en los órganos equivalentes a nivel clínico, asociado a cambios en volumen de sangre en las zonas. En solo unos minutos la termografía es capaz de captar estas diferencias entre zonas alteradas y zonas normales de la piel y extraer diferencias que ayuden al filtrado inicial en la detección de patologías. (Salazar et al., 2015)

La medición de la termografía en la configuración clínica se puede realizar en un lugar determinado o sobre un área de interés. La teletermografía de la cara en sujetos normales ha demostrado que los hombres tienen temperaturas más altas que las mujeres. La afirmación anterior se fundamenta en que los hombres tienen mayor metabolismo basal que las mujeres y su piel disipa más calor por unidad de área de superficie corporal. Del mismo modo, también pueden ocurrir variaciones de edad y etnia en la temperatura facial. (Mouli et al., 2012)

De acuerdo con un sistema reciente de clasificación del $\Delta \mathrm{T}$ (variación de temperatura) se utilizaron teletermógrafos para mediciones del área facial, clasificando como 'normal' cuando los valores $\Delta \mathrm{T}$ del área anatómica variaba de $0.0 \mathrm{a} \pm 0.25^{\circ} \mathrm{C}$, 'caliente' cuando el área $\Delta \mathrm{T}$ es $>-0.35^{\circ} \mathrm{C}$ y 'frío' cuando el área $\Delta \mathrm{T}$ era $<+0.35^{\circ} \mathrm{C}$. Cuando un valor $\Delta \mathrm{T}$ del área anatómica seleccionada es $\pm\left(0.26-0.35^{\circ} \mathrm{C}\right)$, los datos se clasifican como 'equívocos'.

Los termógrafos calientes tenían el diagnóstico clínico de:

1. Dolor mediado por nervios periféricos.

2. Dolor simpático.

3. Sinusitis maxilar.

4. Artropatía de la articulación temporomandibular (ATM).

Se vieron termografías frías con diagnóstico de dolor simpático independiente y nervio periférico. (Lakshmi et al., 2018), (Mouli et al., 2012)

En trastornos de la ATM:

El examen normal de ATM utilizando termografía había mostrado patrones térmicos simétricos con una media $\Delta \mathrm{T}$ valores de $0.10 \mathrm{C}$. Por otro lado, pacientes afectados con trastorno interno y ATM la osteoartritis mostró valores de $\Delta \mathrm{T}$ de $+0.40 \mathrm{C}$. (Lakshmi et al., 2018) 
La aplicación de la TIR para el diagnóstico de la patología de la ATM ya se ha abordado en numerosas publicaciones. Fikackova y Ekberg (2004) valoraron positivamente los resultados obtenidos por la TIR como herramienta diagnóstica en acasos de artralgia de la ATM. (Citado en Hidalgo et al., 2014)

En el año 2013 se estudió la exactitud y la fiabilidad de la TIR en el diagnóstico de dolor en mujeres con disfunción temporomandibular, y se llegó a la conclusión de que la prueba tiene una excelente fiabilidad interevaluadora, pero una baja precisión, en el diagnóstico del dolor de origen miógeno en mujeres con disfunción temporomandibular. También se analizó la temperatura central de los músculos implicados en la masticación (masetero, temporal) y se concluyó que este dato no ofrece una precisión adecuada para el diagnóstico de la disfunción temporomandibular de origen miógeno. (DibaiFilh, Packer, Costa\&Rodrigues-Bigaton, 2013)

Por su parte, Lobo Sanz concluye en su estudio que, considerando los resultados obtenidos y debido al desarrollo de la TIR en los últimos años y a la aparición de cámaras termográficas digitales de alta precisión, parece viable el estudio y el desarrollo de esta técnica en Odontología para el diagnóstico de trastornos musculo esqueléticos de disfunción temporomandibular. (Hidalgo et al., 2014), (Lobo, 2013)

En la evaluación de la vitalidad pulpar:

La vitalidad de la pulpa está bajo investigación. El concepto básico es que el diente que tiene un tejido pulpar vital es de temperatura más cálida en comparación con el diente sin suministro de sangre. Los dientes no vitales fueron más lentos para recalentarse en comparación con los dientes vitales. La limitación de este procedimiento es que los dientes deben aislarse con dique de goma. El procedimiento es complejo y requiere que los sujetos descansen una hora antes de la prueba. (Lakshmi et al., 2018)

En la cuantificación térmica de la pulpa:

El tejido pulpar dental está expuesto a una variedad de lesiones térmicas durante varias modalidades de tratamiento dental.

Últimamente para la desunión de brackets de ortodoncia, se utiliza ampliamente el método de desunión electrotérmica (ETD). Aunque la técnica tiene más ventajas que el método mecánico convencional puede plantear serios daños térmicos a la pulpa. (Mouli et al., 2012)

En la evaluación del déficit del nervio alveolar inferior:

A lo largo de los años, numerosos estudios han demostrado que la técnica de imagen térmica puede desempeñar un papel vital en la evaluación efectiva del déficit del nervio alveolar inferior. Gratt y sus colegas en 1994 declararon que los pacientes con déficit del nervio alveolar inferior cuando se examinaron mostraron valores de $\Delta \mathrm{T}$ de $+0.50 \mathrm{C}$ en el 
lado afectado mientras que los sujetos sin déficit del nervio alveolar inferior mostraron un valor de $\Delta \mathrm{T}$ térmico simétrico de $+0.10 \mathrm{C}$. (Gratt, Sickles, \&Shetty, 1994)

Detección de herpes labial en fase prodrómica:

Durante la fase prodrómica, todos los pacientes mostraron un aumento de temperatura siendo $1.1^{\circ} \mathrm{C} \pm 0.3^{\circ} \mathrm{C}$ sobre un área termográfica positiva media de $126 \mathrm{~mm} 2 \pm 34 \mathrm{~mm}$, incluso cuando el paciente estaba asintomático.

Después de 72 horas de tratamiento con crema de Aciclovir, la mayoría de los pacientes volvieron a la normalidad al realizársele el mismo estudio termográfico sin evidencia de infección. (Sikdar et al., 2010)

Aplicaciones adicionales de teletermografía en Odontología:

1. Diagnóstico de los trastornos óseos y nerviosos/ Evaluación de trastornos del cráneo mandibular.

2. Dolor articular en artritis, osteoartritis, artritis reumatoide.

3. Dolor muscular, reacciones hiper o hipotónicas.

4. Seguimiento de tratamientos endodónticos.

5. Reacciones tisulares a nuevos materiales dentales.

6. Diagnóstico de cualquier tipo de inflamación maxilofacial.

7. Periodontitis crónica y aguda.

8. Enfermedad sinusal

9. Cánceres en territorio maxilofacial

10. Síndrome de dolor miofascial

11. Neuralgia.

12. Detección de la enfermedad oclusalcarotídea. (Mouli et al.,2012)(Sikdar et al., 2010)

En 1998, se desarrolló un sistema que utiliza la luz infrarroja para detectar caries basándose en la diferencia de fluorescencia entre el esmalte sano y el desmineralizado. Este sistema nos ayuda a detectar si la lesión está afectando solo el esmalte o también la dentina, debido a la diferencia de la luz emitida y localización de la misma. (Hibst\&Gall,1998)

Pérez (2004) expresa que "con esta técnica se detecta una lesión inicial de caries con gran precisión y reproducibilidad, en comparación con las técnicas convencionales, sin embargo, no parece ser válida para el diagnóstico de lesiones de caries oclusal en molares deciduos".

En la actualidad, y en el campo de las ciencias de la salud, hay una serie de patologías que se han estudiado gracias a la termografía y para las que esta técnica aporta datos de diagnóstico relevantes. Las más útiles para la investigación son las relacionadas con alteraciones en los sistemas corporales encargados de mantener la homeostasis, y que se refieren a que variaciones de unos pocos grados que indican una disfunción en el cuerpo 
donde los procesos químicos del mismo están sufriendo alteraciones.(Jones 1998)Salazar et al.(2015) apuntan que las aplicaciones más directas de la termografía a la medicina en la actualidad se dirigen hacia la evaluación del riesgo, a añadir información diagnóstica, a la monitorización de tratamientos y a los indicadores de pronóstico.

Salazar et al. (2015) señala que "la técnica tiene un coste elevado, y aunque con el paso de los años se ha ido abaratando, aún no está disponible en la mayoría de los centros de investigación especializados". Su elevado precio no obstaculiza las ventajas que experimenta sobre otros métodos tradicionales por lo que resulta de gran interés socializarlos con los niveles de pregrado y el resto de los especialistas de nuestro campo.

De acuerdo a lo que se ha planteado, varios han sido los artículos en los que se ha demostrado que la termografía no solo es un método rápido y no invasivo, sino que su empleo respecto a otras técnicas y métodos ya instaurados puede ser de gran utilidad en la conservación de la salud de las personas y es preciso su conocimiento para su aplicación. (González, Ruiz, Benítez \&Figueroa, 2019)

En relación a lo revisado, las autoras consideran que constituye una herramienta novedosa que debería ser conocida por nuestros especialistas ya que ofrece múltiples beneficios en el diagnóstico de enfermedades.

Las autoras consideran que la termografía se presenta como una de las mejores pruebas a la hora de detectar diferentes enfermedades, situándose a la altura de las radiografías y la MRI (Imagen por resonancia magnética).

\section{Conclusiones}

- La termografía es una opción útil para complementar el diagnóstico de patologías bucales que implican un cambio de temperatura en la región orofacial.

- Su aplicación en el diagnóstico de patologías en la ATM ha tenido gran importancia.

- Su uso en nuestro campo de trabajo debe ser conocido por los que pertenecen a los niveles de pregrado y también por el resto de los profesionales estomatólogos.

\section{Referencias bibliográficas}

DibaiFilho, A.V., Packer, A.C., Costa, A.C.,\&Rodrigues-Bigaton, D. (2013) Accuracy of infraredthermography of themasticatorymusclesforthe diagnosis of myogenoustemporomandibulardisorder. J ManipulativePhysiolTher, 36(4) ,245-52. Recuperado de:http://dx.doi.org/10.1016/j.jmpt.2013.04.007

Fikackova, H. \&Ekberg, E.(2004)Can infraredthermography be a diagnostictoolforarthralgia of thetemporomandibularjoint? Oral surgery, oral medicine, oral pathology, oral radiology and endodontics, 98(6), 643-650. 
González, Y., Ruiz, J.E., Benítez, M., \&Figueroa, D.V. (2019). La Termografía y su Aplicabilidad en la Medicina. En H.Sánchez-Toledo (Presidencia), Por la sostenibilidad y la calidad de los procederes tecnológicos en salud. IV Congreso Internacional Tecnología y Salud 2019,La Habana, Cuba.

Gratt, B.M., Sickles, E.A., \&Shetty, V. (1994) Termografía para la evaluación clínica del déficit del nervio alveolar inferior: un estudio piloto. J Dolor Orofacial, 8, 369-374.

Hibst, R.,\&Gall, R. (1998) Development of a diode laser-basedfluorescence caries detector. Caries Res, 32, 294.

Hidalgo Salvador, E., Álvarez González, F., \& Salvador Luna, A. (2014). Application of Infraredthermography in legal medicine: isit a valid test foranobjectiveassessment of painfulsyndromes? Temporomandibulardisorder. Cuadernos de Medicina Forense, 20(2-3), 77-84. https://dx.doi.org/10.4321/S1135-76062014000200002

Jones, B. F. (1998). A reappraisal of the use of infraredthermalimageanalysis in medicine. Medical Imaging, IEEE Transactionson, 17(6), 1019-1027.

Lakshmi, P. S., Kiran M. S., \&Joy E., T. (2018). A REVIEW ON THERMOGRAPHY IN DENTISTRY. Journal of Evolution of Medical and Dental Sciences, 7(10), 12891293.

Leal Brioschi, M. (2006). Anatomia e fisiología Termográfica: Sociedade Brasileira de Termología PanamericanThermologySociety.

Lobo, A.M. (2013) Estudio previo experimental comparativo sobre el uso de boquillas personalizadas frente a boquillas estándar en la práctica del buceo con escafandra autónoma para la prevención del síndrome bucal del buceador. Trabajo para la obtención del título de Máster Oficial en Ciencias Odontológicas. Universidad Complutense de Madrid, Madrid, España.

Mouli, Dr\&Kumar, Sachidanandan\&Balasubramani, Senthil\&Parthiban, Sree\&Malarvizhi, Dr\&Karthik, Dr. (2012). Application of Thermography in Dentistry-A Review. IOSR Journal of Dental and Medical Sciences, 1, 39-43. Recuperado de: https://www.researchgate.net/publication/272715778_Application_of_Thermograp hy_in_Dentistry-A_Review

Pérez, A. (2004). Capacidad diagnóstica de la fluorescencia láser para el diagnóstico +de caries oclusal en dientes deciduos. Revista Estomatológica Herediana, 4(1-2), 5-11.

Presídeo, L., Wanerly, F., \& Medrano, A. (2016). O USO DA TERMOGRAFIA INFRAVERMELHA NA ODONTOLOGIA E SUAS ESPECIALIDADES. Revista Bahiana de Odontologia, 7(2), 155-165.Recuperado de: https://www.researchgate.net/publication/304992785_O_USO_DA_TERMOGRAF IA_INFRAVERMELHA_NA_ODONTOLOGIA_E_SUAS_ESPECIALIDADES_ UMA_REVISAO_SISTEMATICA 
Ring, E.F.J.(2007). Thehistoricaldevelopment of temperaturemeasurement in medicine. Infaredphysics\&Technology,49,297-301.

Salazar, E., Domínguez, E., Iborra, O., de Córdoba, M.J.\& Gómez, E. (2015).Breve historia y áreas de aplicación de la Termografía. En Gómez, E., Salazar, E., Domínguez, E., Iborra, O., de la Funte, J., \& de Córdoba, M.J. (Ed.), NeuroTermografía y Termografía Psicosomática (pp.35-50). Granada, España: Ediciones Fundación Internacional Artecitta. Recuperado de: https://www.researchgate.net/profile/Juanma_De_La_Fuente/publication/27689390 6_Neurotermografia_y_termografia_psicosomatica/links/555c626708ae91e75e76f2 7d/Neurotermografia-y-termografia-psicosomatica.pdf

Sikdar, S.D., Khandelwal, A., Ghom, S., Diwan, R.,\&Debta, F.M.(2010). Thermography: A new Diagnosis Tool in Dentistry. Journal of IndianAcademy of Oral Medicine \&Radiology, 22 (4), 206-210. 
PARA CITAR EL ARTÍCULO INDEXADO.

de León Ramírez, L. L., Garay Crespo, M. I., de León Ramírez, L. R., \& Magariño Abreus, L. (2021). Termografía, una herramienta novedosa en la práctica estomatológica. Anatomía $\quad$ Digital, $\quad 4(2), \quad 6-16$. https://doi.org/10.33262/anatomiadigital.v4i2.1613

\section{Ciencia \\ LDigital}

El artículo que se publica es de exclusiva responsabilidad de los autores y no necesariamente reflejan el pensamiento de la Revista Anatomía Digital.

El artículo queda en propiedad de la revista y, por tanto, su publicación parcial y/o total en otro medio tiene que ser autorizado por el director de la Revista Anatomía Digital.
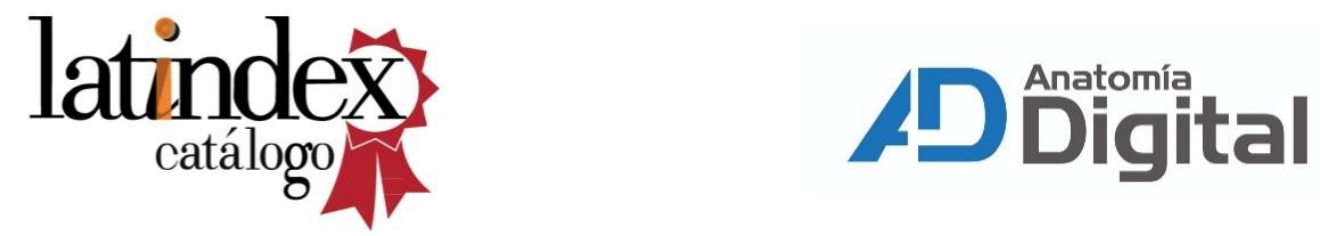